\title{
Zirconia sulfatada como un catalizador para la síntesis de biodiesel
}

\author{
J. S. Acevedo ${ }^{\text {a }}$ F. J. Garza-Méndez ${ }^{\text {a* }}$, E. M. Sánchez-Cervantes ${ }^{a}$, J. Aguirre-Espinosa ${ }^{\text {a }}$ \\ ${ }^{a}$ Universidad Autónoma de Nuevo León, UANL, Facultad de Ciencias Químicas, Av. Universidad S/N Ciudad Universitaria San Nicolás \\ de los Garza Nuevo León CP 66451 México, Tel (81) 83294000 \\ ${ }^{*}$ E-mail: francisco.garzamd@uanl.edu.mx
}

Recibido: 25 septiembre 2013, Aceptado 14 noviembre 2013

\begin{abstract}
Resumen
En este trabajo se sintetizaron nanopartículas de zirconia vía microondas, éstas se utilizaron como soporte de catalizadores sólidos. Se preparó hidróxido de zirconio por el método sol-gel a partir de propóxido de zirconio e hidróxido de amonio, una vez obtenido el gel se secó y se sometió a calentamiento por microondas y un enfriamiento rápido. La zirconia se sulfató con una solución de ácido sulfúrico y se utilizó como catalizador en la producción de biodiesel observando su actividad catalítica.

Las nanopartículas de zirconia obtenidas son de un tamaño de 50 a $60 \mathrm{~nm}$ con una mezcla de fases tetragonal y monoclínica, predominando la tetragonal, y un área superficial de $180.6 \mathrm{~m}^{2} / \mathrm{g}$, esto se comprobó mediante difracción de rayos-X en polvos (DRX), microscopía electrónica de barrido (MEB) y la técnica Brunauer-Emmett-Teller (BET), uno de los logros principales fue que no se modificó el microondas y hubo mucho menos consumo de energía comparado con el método tradicional de calcinación, finalmente, se observó la conversión de aceite vegetal utilizado a biodiesel mediante la cromatografía de gases (CG), los rendimientos obtenidos son comparables con la síntesis de catálisis básica homogénea.
\end{abstract}

Palabras clave: Biodiesel, zirconia, microondas.

\section{Introducción}

El biodiesel es un combustible no derivado del petróleo basado en ésteres de alquilo derivados de cualquiera de las transesterificaciones de los triglicéridos o la esterificación de ácidos grasos libres con alcoholes de baja masa molecular [1]. Las propiedades de flujo y la combustión de biodiesel son similares a las del diesel obtenido del petróleo y, por lo tanto, puede ser utilizado como su sustituto o la mezcla de ambos [2]. Como punto de comparación, el biodiesel puro (B100) libera aproximadamente un $90 \%$ de la energía con respecto al diesel normal, y por lo tanto, su rendimiento esperado en el motor es casi el mismo en términos de torque y potencia. El biodiesel, sin embargo, se puede sintetizar a partir de aceite reciclado comúnmente, es biodegradable y no tóxico, y tiene un punto de inflamación más alto que el diesel normal. Además, el biodiesel aumenta la lubricación (incluso en mezclas de sólo $3 \%$ o menos), lo que prolonga la vida del motor y reduce la frecuencia de sustitución de partes de éste. Otra ventaja significativa del biodiesel es su perfil de baja emisión y su contenido de oxígeno de 10$11 \%$. El biodiesel se conoce como el biocombustible amigable con el medio ambiente, ya que proporciona un medio para reciclar el dióxido de carbono. En otras palabras, el biodiesel no contribuye al calentamiento global. Las normas ASTM para el biodiesel presentan características que son comparables con el combustible diesel tradicional [3]. La Tabla 1 resume los perfiles de emisión típicos de biodiesel y una de sus mezclas, B20, que consta de $20 \%$ de biodiesel y $80 \%$ diesel, utilizando las emisiones del diesel derivado del petróleo como referencia.
La transesterificación requiere un catalizador con el fin de obtener rendimientos razonables de conversión. La naturaleza del catalizador es fundamental, ya que determina los límites de composición que el material de alimentación debe cumplir.

Actualmente, la mayoría del biodiesel se prepara utilizando catalizadores alcalinos, tales como metóxidos e hidróxidos de sodio y potasio. Industrialmente, $\mathrm{NaOH}$ y $\mathrm{KOH}$ se prefieren debido a su amplia disponibilidad y bajo costo [4]. No obstante, desde un punto de vista químico, las especies activas con ambos tipos de catalizadores son iones metóxido, éstos son nucleófilos fuertes y atacan el grupo carbonilo en las moléculas de glicéridos para producir ésteres de alquilo. En general el proceso tiene serias limitaciones que se traducen en altos costos de producción. Por otra parte, el alcohol y el catalizador deben cumplir con las rigurosas especificaciones también, deben ser anhidros, ya que la presencia de agua en el material de alimentación promueve la hidrólisis de los ésteres de alquilo de ácidos grasos libres, por lo anterior se requiere el uso de aceites vegetales altamente refinados cuyo precio puede ser del 60 al $75 \%$ del costo final del biodiesel [5].

El biodiesel producido como tal no es tan amigable con el medio ambiente como podría parecer en un primer término, ya que se utilizan campos para producir semillas específicamente para los aceites refinados, en vez de usar dichos campos para producir alimentos, es por ello que algunos investigadores buscan producir diesel a partir de reciclar aceites comestibles usados y grasa animal. 
Debido a su precio, el uso de materias primas de grasa de desecho se ha propuesto como una manera de reducir los costos de producción de biodiesel, algunas plantas en los Estados Unidos ya están produciendo biodiesel de grasa amarilla [6], pero también las grasas marrones de los filtros de los centros comerciales pueden ser utilizadas. Las materias primas de bajo costo deben experimentar alguna forma de tratamiento antes de que puedan ser utilizadas para la producción de biodiesel, la materia particulada se separa con filtros de celulosa, el agua se separa por gravedad y mediante destilación se desnaturaliza y degradan las proteínas residuales en la grasa, así como azúcares y alcoholes producidos durante el almacenamiento [7].

La temperatura juega un papel importante en la síntesis del biodiesel catalizada por ácidos. A temperaturas más altas, la separación de fases disminuye y las constantes de velocidad aumentan, redundando en tiempos de reacción sustancialmente cortos. Normalmente, las concentraciones de catalizador en la mezcla de reacción varían entre 1 y $5 \%$ en peso [8].

Las zeolitas de poro grande se han utilizado con cierto éxito en la esterificación de ácidos grasos en medio ácido; sin embargo, siempre ocurre que se originan subproductos no deseados debido a la alta temperatura de la reacción [9].

Por otra parte, la zirconia se ha empleado como catalizador en un sinnúmero de reacciones químicas, en este trabajo se propone el uso de zirconia como un soporte para catalizadores ácidos [10]

En el 2006 Tyagi et al [11] sintetizaron nanopartículas de zirconia que tienen fase cristalina predominante tetragonal usando el método sol-gel $\mathrm{y}$ técnicas convencionales de precipitación de hidróxido de zirconio obtenido por la hidrólisis de propóxido de zirconio y oxicloruro de zirconio como precursores. Se observó que el secado térmico de gel de hidróxido de zirconio en un horno $\left(110^{\circ} \mathrm{C}, 12 \mathrm{~h}\right)$ dio resultados de tamaño de cristales inferiores (11-13 nm) en comparación con el secado a vacío $\left(50 \mathrm{mbar}, 70^{\circ} \mathrm{C}\right)$, que muestra mayor tamaño de los cristales (20 -21 nm) durante ambas técnicas, sol-gel y la síntesis de precipitación convencional.

En el 2010 Lee et al [12] utilizaron el dióxido de carbono supercrítico $(\mathrm{scCO} 2)$ líquido como disolvente verde que puede reducir considerablemente el uso de disolventes orgánicos. Los grupos de zirconia nanoporosa $(\mathrm{ZrO} 2)$ se sintetizaron de una microemulsión $[\mathrm{Zr} 4+]$ (aq) / scCO2 usando una solución de hidracina como agente de precipitación.

En el 2011 Kazemi et al [13] sintetizaron nanopartículas de zirconia $(\mathrm{ZrO} 2)$ utilizando sacarosa y fructosa como agentes quelantes del hidróxido de zirconio. En esta investigación se observó que los agentes quelantes de sacarosa y fructosa dan como resultado una fase tetragonal de la zirconia y un tamaño de partícula menor al que se obtiene con el método convencional utilizando así menores temperaturas.

En el 2012 Thitsartarn et al [14] sintetizaron zirconia sulfatada soportada en SBA-15 utilizando un método post-síntesis y fue comparada con la zirconia sulfatada convencional en términos de actividad catalítica en la transesterificación de aceite de palma. Los catalizadores P-SZS podría conservar la estructura mesoporosa ordenada hexagonal incluso con concentraciones de zirconia altos, y tenían mayor acidez que la zirconia sulfatada. Como resultado, estos catalizadores mostraron mayor actividad catalítica que el catalizador de zirconia sufatada convencional para la transesterificación de aceite de palma con metanol.

En el 2012 Tyagi et al [15] evaluaron la actividad catalítica de la zirconia sulfatada nanocristalina, preparada por método sol-gel para la esterificación de ácido caprílico con diferentes alcoholes. La concentración más baja de catalizador $(0,5 \%$ en peso) exhibió $96-98 \%$ de conversión del ácido caprílico con metanol y $100 \%$ de selectividad para caprilato de metilo a $60{ }^{\circ} \mathrm{C}$. La conversión se redujo al aumentar la masa molecular de los alcoholes (etanol, n-propanol y nbutanol a $\left.60{ }^{\circ} \mathrm{C}\right)$, pero aumentó significativamente $(91$ $98 \%$ ) al aumentar la temperatura de reacción.

Por otra parte, en 2009 Cao et al sintetizaron mediante microondas diversos materiales mesoporosos [16], principalmente óxido de silicio y alúmina. Este método tiene la ventaja de la nucleación homogénea.

\section{Parte experimental}

\subsection{Síntesis de zirconia}

Para sintetizar la zirconia se colocaron $25 \mathrm{~mL}$ de propóxido de zirconio en un vaso de precipitado, posteriormente se añadió gota a gota una solución de hidróxido de amonio al $25 \%$ hasta que obtener un $\mathrm{pH}$ entre 9 y 10. Una vez añadido el hidróxido de amonio se agitó durante una hora hasta obtener un gel de aspecto transparente e incoloro. El gel obtenido se secó en una estufa a $101{ }^{\circ} \mathrm{C}$ por $12 \mathrm{~h}$. Una vez seco el gel se colocó 1 gramo en un crisol de porcelana, dicho crisol se ubica encima de un crisol de alúmina que contiene una cama de grafito molido y se introduce en el microondas, el grafito en polvo absorbe microondas y al calentarse por conducción calienta el hidróxido de zirconio, el cual, una vez alcanzada cierta temperatura absorbe microondas calentándose homogéneamente. Este proceso se llevó a cabo a diferentes intervalos de tiempo desde 30 hasta 90 segundos, inmediatamente se saca el crisol que contiene el producto y se enfría en un recipiente de aluminio que yace sobre nitrógeno líquido a presión atmosférica.

\subsection{Sulfatación de la zirconia}

Se coloca un gramo de zirconia en un vaso de precipitado y se añaden $15 \mathrm{~mL}$ de una solución de ácido sulfúrico de $0.5 \mathrm{M}$ a $1.5 \mathrm{M}$ y se agita constantemente durante $12 \mathrm{~h}$. Finalmente se separa la zirconia mediante filtración y se seca en un horno a $110^{\circ} \mathrm{C}$ durante $3 \mathrm{~h}$.

\subsection{Producción de biodiesel}

Para llevar a cabo la síntesis del biodiesel se colocan en un matraz bola $50 \mathrm{~mL}$ de aceite vegetal reciclado, el cual es previamente filtrado y desnaturalizado, $100 \mathrm{~mL}$ de etanol y se le añade $1.0 \%$ en masa del catalizador. El sistema se deja reaccionar entre 60 y $80{ }^{\circ} \mathrm{C}$ durante $2 \mathrm{~h}$, siempre en agitación constante y reflujo. Terminada la reacción se separa el catalizador mediante filtración y se 
seca a $110^{\circ} \mathrm{C}$ durante $3 \mathrm{~h}$. Por otra parte, las fases son separadas en un embudo de separación, dejándolas reposar $24 \mathrm{~h}$.

\subsection{Difracción de rayos-X}

Mediante difracción de rayos-X en polvos se caracterizaron tanto la zirconia sintetizada por microondas así como la zirconia después de la sulfatación. Todas las difracciones se realizaron en las siguientes condiciones: $2 \theta$ entre $5^{\circ}$ y $90^{\circ}$, con un paso de $0.05^{\circ}$, y temperatura ambiente $\left(\sim 25^{\circ} \mathrm{C}\right)$.

\section{5. Área superficial}

El área superficial y el tamaño de partícula se calcularon empleando la técnica de BET mediante ciclos de adsorción-desorción de nitrógeno. Se utilizó un equipo Quantachrome Instruments modelo Autosorb-1.

\subsection{El microscopio electrónico de barrido (MEB)}

Para observar las partículas de zirconia se utilizó microscopía electrónica de barrido de alta resolución en la modalidad de electrones secundarios.

\section{Resultados y discusión}

\subsection{1. Área superficial y tamaño de poro}

La Figura 1 presenta un análisis BJH (algoritmo Barret-Joyner-Halenda) del tamaño de poro y el análisis volumétrico, basado en la rama de desorción obtenido directamente del ordenador del equipo, se muestra una distribución muy cerrada del tamaño de poro. Tras el análisis, se calculó un diámetro de poro promedio de 30 $\mathrm{nm}$ y un área superficial promedio de $180.6 \mathrm{~m}^{2} / \mathrm{g}$, de 10 cual se concluye que la zirconia obtenida por microondas convencional es un material mesoporoso.

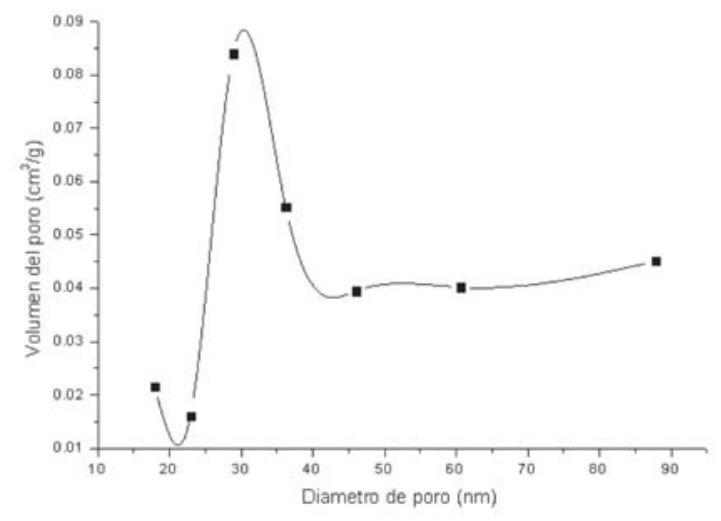

Figura 1. Distribución del tamaño de poro para la zirconia vía microondas, curva obtenida por análisis $\mathrm{BJH}$.

La Figura 2 muestra la comparación de la distribución del tamaño de poro de la zirconia sulfatada y sin sulfatar, se puede observar que hay un ligero crecimiento en el tamaño de poro pero se sigue manteniendo en el rango de un material mesoporoso, sin embargo al tener un mayor tamaño de poro reduce su área superficial lo cual es comprobado con el análisis BET obteniendo un área superficial de $132.2 \mathrm{~m}^{2} / \mathrm{g}$.

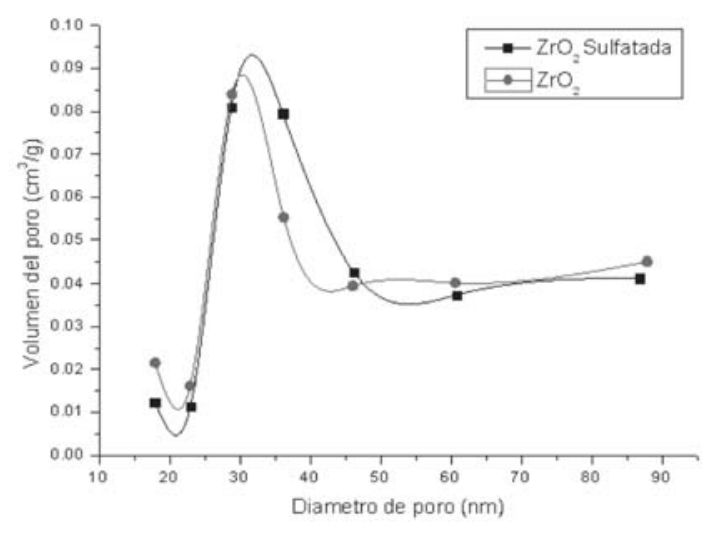

Figura 2. Distribución del tamaño de poro para la zirconia vía microondas sulfatada, curva obtenida por análisis BJH.

Al observar cómo se reduce el área superficial debido a la sulfatación se realizaron sulfataciones con distinta concentración para observar el efecto que tiene en el área superficial, los resultados se muestran a continuación en la Tabla 2.

Tabla 2. Área superficial a diferentes concentraciones de acidez.

\begin{tabular}{c|c} 
Acidez $(\% \mathrm{w} / \mathrm{w})$ & Área superficial $\left(\mathrm{m}^{2} / \mathrm{g}\right)$ \\
\hline 1.6 & 132.2 \\
2.1 & 118.4 \\
2.7 & 103.8 \\
\hline
\end{tabular}

Como se puede observar ocurre una disminución en el área superficial de la zirconia al tener mayor acidez, este es un factor importante ya que el área superficial desempeña un papel importante en los catalizadores pero esta disminución de área es nivelado al tener mayor acidez, ya que la acidez cataliza la reacción para obtener biodiesel, esto se confirma en los resultados en la síntesis de biodiesel.

\subsubsection{Difracción de rayos-X}

A continuación se mostrarán los resultados obtenidos en la difracción de rayos-X, para poder identificar los picos se utilizó como base la Figura 3 la cual presenta los patrones característicos de cada estructura que puede presentar la zirconia [32] 


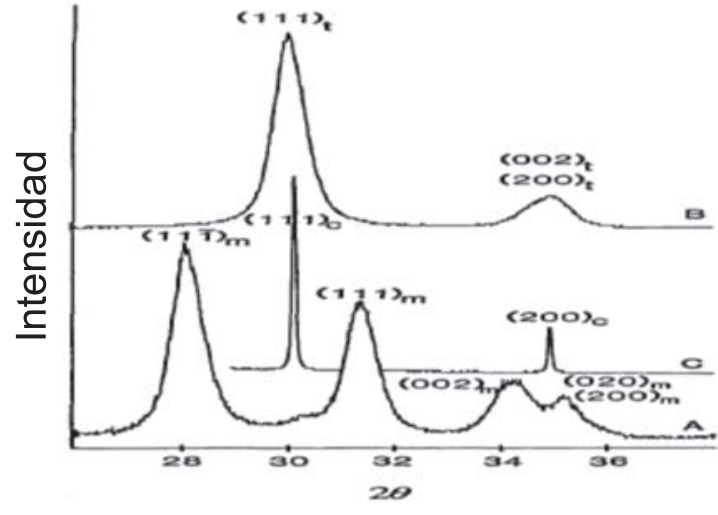

Figura 3. Patrones de difracción de rayos $\mathrm{X}$ típicos de la zirconia. (A) $100 \%$ zirconia monoclínica, (B) $100 \%$ zirconia tetragonal y (C) 100\% zirconia cúbica.

De la Figura 4 a la Figura 6 se muestran los difractogramas de la zirconia sintetizada vía microondas. Se registraron mezcla de fases, siendo la fase predominante la tetragonal, esto se traduce en un gran logro tomando en cuenta dos aspectos importantes en la síntesis: (i) la atmósfera empleada fue aire y (ii) se controló sólo la potencia $1500 \mathrm{~W}$ y la frecuencia de 2.45 $\mathrm{GHz}$.

En la Figura 4 se puede observar que se obtiene zirconia en fase tetragonal por los picos característicos en los ángulos de difracción aproximadamente en $30^{\circ}$, $35^{\circ}$ y $60^{\circ}$, con forme va incrementando el tiempo de reacción se puede observar una aparición de un pico a un ángulo de difracción $\sim 28^{\circ}$ (Figura 5), este pico es característico de una fase monoclínica y se ve como incremente al avanzar aún más el tiempo de reacción (Figura 6) pero al mismo tiempo se observa como ocurre un desdoblamiento de los picos que se encuentran a $\sim 35^{\circ}$ y a $\sim 60^{\circ}$, esto indica la formación de una fase tetragonal más definida sin embargo se comienza a formar una fase monoclínica en el sistema la cual puede ser una de las causas de la morfología final de la zirconia y puede afectar en sus propiedades catalíticas en la producción de biodiesel.

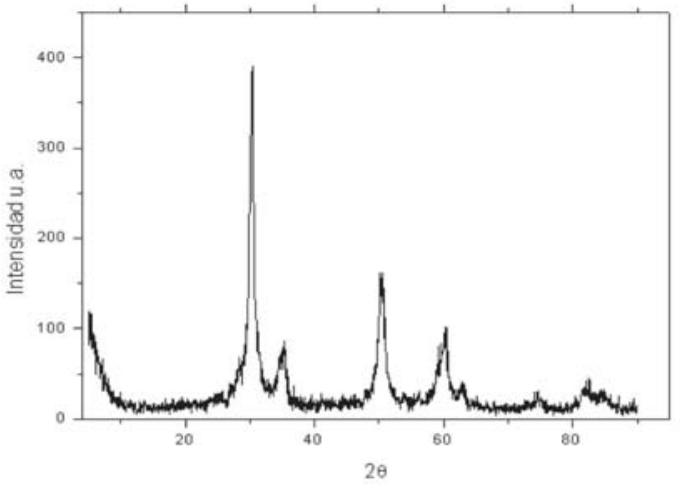

Figura 4. Difracción de rayos- $\mathrm{X}$ en polvo de la zirconia a $30 \mathrm{~s}$ de calentamiento en microondas.

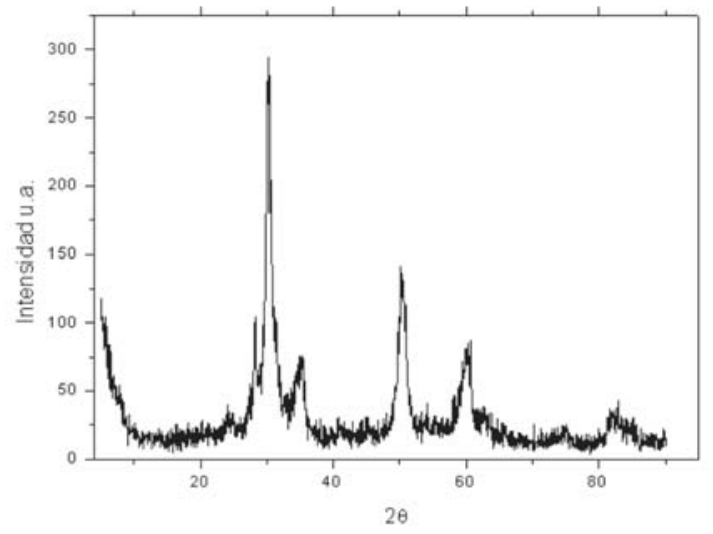

Figura 5. Difracción de rayos- $X$ en polvo de la zirconia a $60 \mathrm{~s}$ de calentamiento en microondas.

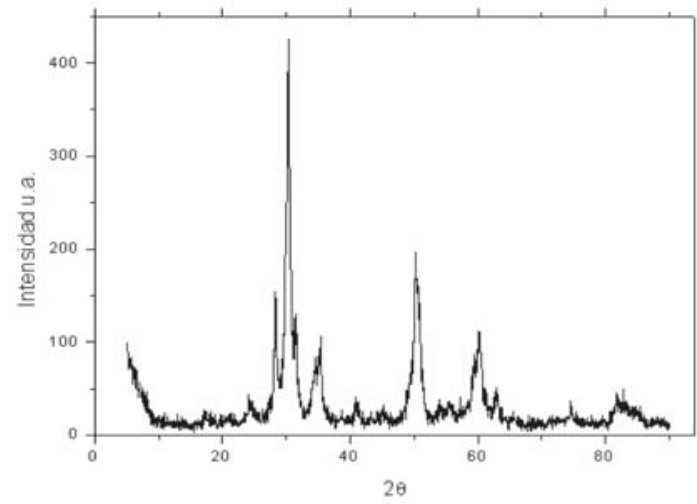

Figura 6. Difracción de rayos-X en polvo de la zirconia a $90 \mathrm{~s}$ de calentamiento en microondas.

En la Figura 7 se muestran los difractogramas de la zirconia sintetizada vía microondas sulfatada por el método de impregnación, en los difractogramas se puede observar que después de la sulfatación no ocurrió ningún cambio en la estructura de la zirconia, sin embargo esto no confirma que no haya sucedido un cambio en la morfología ya que la sulfatación es un proceso que se da en la superficie de la zirconia y es probable que haya un crecimiento en el tamaño de partícula.

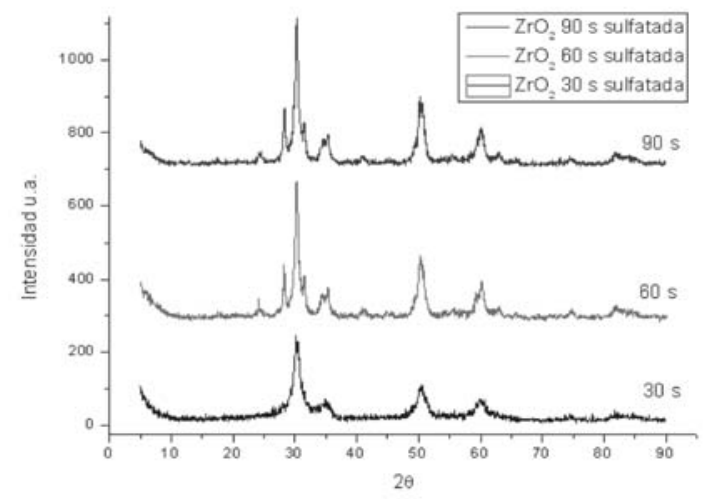

Figura 7. Difractogramas de la zirconia sintetizada vía microondas sulfatada. 
Sabemos que con el tiempo la temperatura que alcanza el sistema va aumentando, si se mantiene en un lapso de tiempo determinado la temperatura incrementará constantemente, pero al fijar un tiempo total de calentamiento y cumplirlo mediante lapsos de calentamiento de tiempo menor se obtiene una estructura tetragonal (Figura 8), ya que, no alcanza la temperatura suficiente para sufrir un cambio de fase pero si la necesaria para obtener una fase definida, en este caso la tetragonal que es la que se obtiene a menores temperaturas a comparación de la monoclínica.

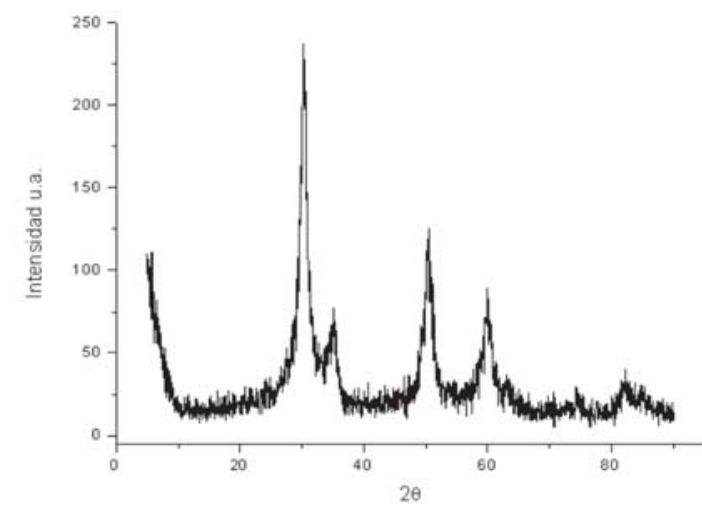

Figura 8. Difractograma de la zirconia sintetizada vía microondas en $120 \mathrm{~s}$ utilizando lapsos de $30 \mathrm{~s}$ de calentamiento.

\subsubsection{Microscopía electrónica de barrido}

La Figura 9 es una muestra de síntesis de la zirconia vía microondas en un lapso de tiempo de 30 a $60 \mathrm{~s}$, la cual está sulfatada. Se empleó el software Gatan MicroGraph para hacer análisis de imágenes, se midió el tamaño de las partículas más grandes, las cuales tienen un tamaño promedio de $24.45 \mu \mathrm{m}$ y una desviación estándar de $4.43 \mu \mathrm{m}$.

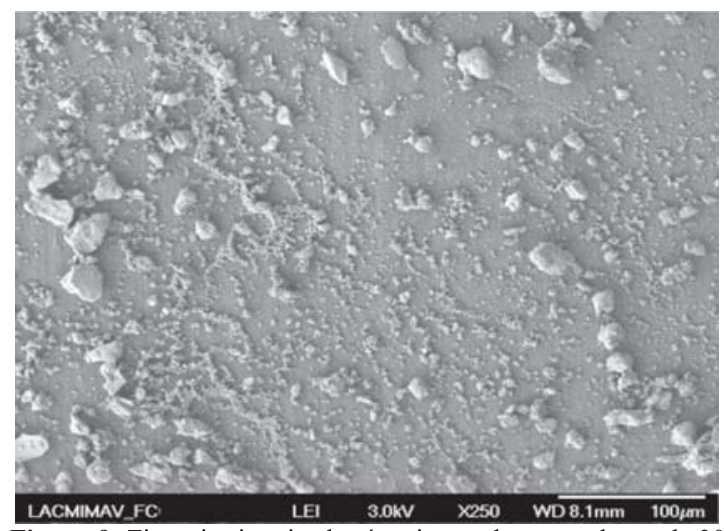

Figura 9. Zirconia sintetizada vía microondas en un lapso de 30 a $60 \mathrm{~s}$ sulfatada, $250 \mathrm{X}$ a $3.0 \mathrm{kV}$.
Al aumentar la magnificación a 5000X, se observa que dichas partículas constan de aglomeraciones de cúmulos de partículas de morfología esférica. La Figura 10 muestra uno de esos cúmulos de pequeñas partículas de morfología esférica. Se observa una distribución bimodal de tamaño de partícula. Para la distribución mayor el tamaño promedio es de $320.27 \mathrm{~nm}$ con una desviación estándar de $36.05 \mathrm{~nm}$. Para las partículas pequeñas el tamaño promedio es de $52.30 \mathrm{~nm}$ y una desviación estándar de $5.31 \mathrm{~nm}$.

La Figura 11 muestra otro de estos cúmulos de nanopartículas esféricas, este mismo cúmulo se observa a $100000 \mathrm{X}$, estas partículas no están sulfatadas, y tienen un tamaño promedio de $64.28 \mathrm{~nm}$ y una desviación estándar de $1.93 \mathrm{~nm}$. Se observa que estos cúmulos constan de nanopartículas esféricas.

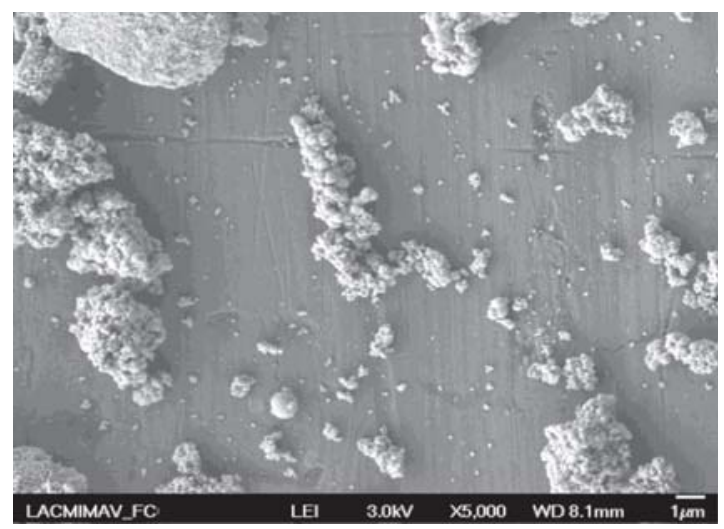

Figura 10. Zirconia sintetizada vía microondas en un lapso de 30 a 60 s sulfatada, $5000 \mathrm{X}$ a $3.0 \mathrm{kV}$.

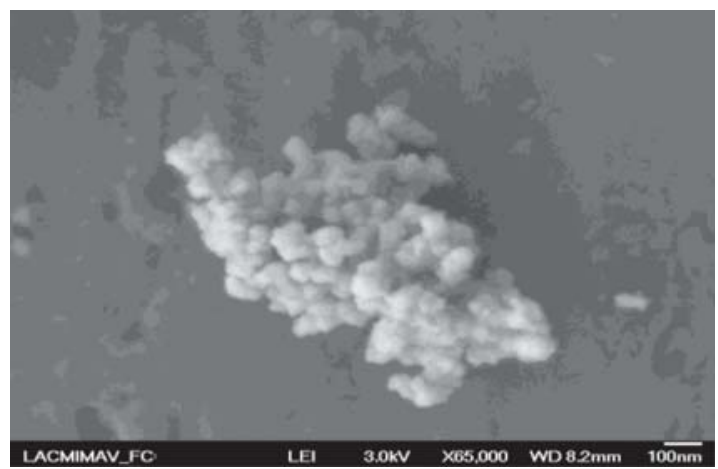

Figura 11. Zirconia sintetizada vía microondas en un lapso de 30 a $60 \mathrm{~s}, 100000 \mathrm{X}$ a $3.0 \mathrm{kV}$.

\subsection{Caracterización del biodiesel}

\subsubsection{Cromatografía de gases}

En la Figura 12 se muestra el cromatograma de aceite del cual se partió para la síntesis de biodiesel, se observa que se mantiene la línea base y no produce señal.

En la Figura 13 se muestra un cromatograma del biodiesel sintetizado utilizando un catalizador básico $(\mathrm{NaOH})$, este biodiesel fue utilizado como estándar para encontrar los tiempos de retención del biodiesel $\mathrm{y}$ 
observar los picos característicos. El primer pico que aparece en el cromatograma es del etanol utilizado en la reacción. Los picos que aparecen a partir del minuto 17 hasta el 22 conforman los esteres sintetizados en la reacción, podemos saber que son los ésteres ya que se inyectó una muestra de aceite y la línea base no muestra señal alguna en el período de análisis, podemos decir que el biodiesel en las condiciones mencionadas anteriormente presenta 4 picos característicos.

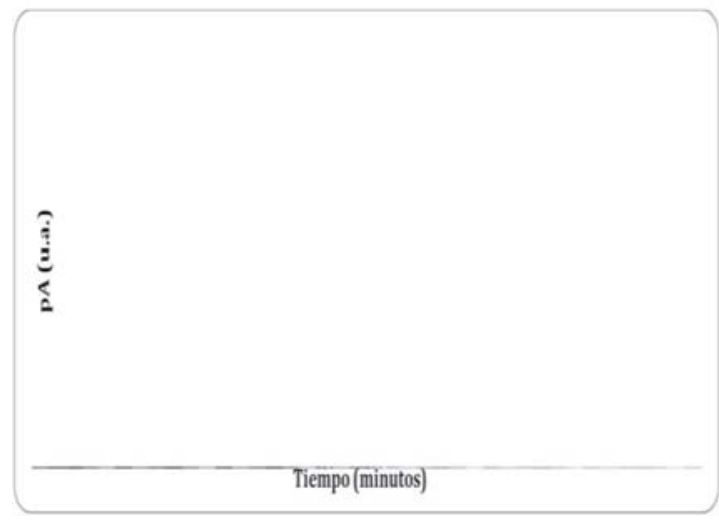

Figura 12. Cromatograma del aceite utilizado en la síntesis de biodiesel.

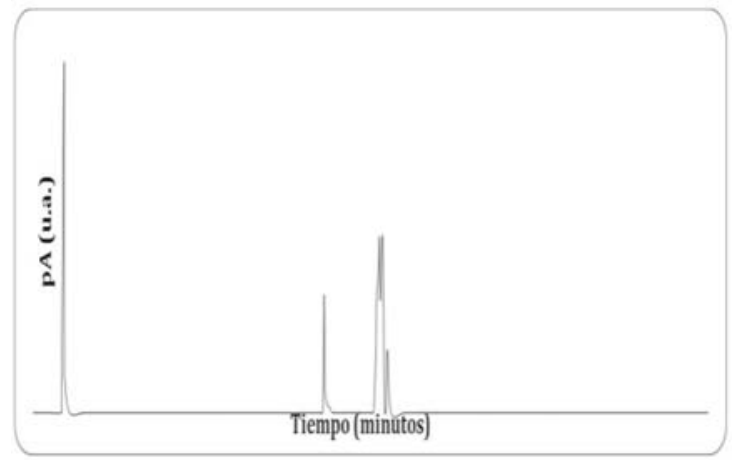

Figura 13. Cromatograma del biodiesel sintetizado con catalizador básico

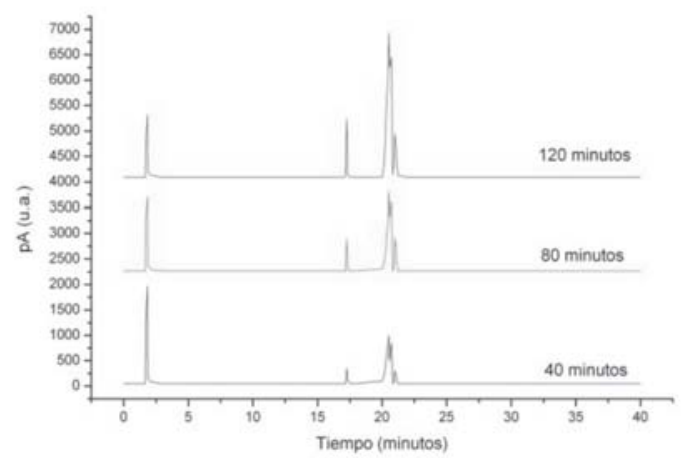

Figura 14. Cromatogramas del seguimiento de la síntesis de biodiesel utilizando la zirconia sulfatada como catalizador.
A lo largo de la reacción del biodiesel se tomaron muestras a distintos lapsos de tiempo $(40,80$ y 120 minutos) y se analizaron para observar cómo progresaba la reacción, en la Figura 14 se muestran los cromatogramas y se puede apreciar claramente como los picos característicos del biodiesel aumentan su intensidad al pasar el tiempo demostrando así que si se logra la transesterificación del aceite utilizando la zirconia sulfatada como catalizador. Además de observar que la conversión aumenta con el tiempo se analizó el porcentaje de humedad y la densidad del biodiesel después de haberle dado un tratamiento de secado y se obtuvo una humedad de $0.08 \%$ y una densidad de 0.884 $\mathrm{g} / \mathrm{cm}^{3}$ las cuales son ligeramente mayores comparadas con el biodiesel sintetizado de manera convencional que tiene una humedad de $0.06 \%$ y una densidad de 0.873 $\mathrm{g} / \mathrm{cm}^{3}$, según la norma americana ASTM el biodiesel debe estar en un rango de humedad máximo al $0.05 \% \mathrm{y}$ una densidad de 0.860 a $0.900 \mathrm{~g} / \mathrm{cm}^{3}$, esta norma establece las especificaciones en las cuales el biodiesel puede ser utilizado como combustible.

En la Tabla 3 se muestran los rendimientos obtenidos de la síntesis de biodiesel durante 2 horas utilizando como catalizador la zirconia y la zirconia sulfatada con diferentes concentraciones de ácido.

Tabla 3. Rendimientos de la síntesis de biodiesel.

\begin{tabular}{c|c}
\hline & Rendimiento (\%) \\
\hline Zirconia & 32.3 \\
Zirconia/SO $/(0.5 \mathrm{M})$ & 88.6 \\
Zirconia/SO & $(1.0 \mathrm{M})$ \\
Zirconia/SO & 84.8 \\
\hline
\end{tabular}

\section{Conclusiones}

Se puede concluir que la zirconia sin sulfatar tiene un rendimiento bajo, mientras que la zirconia sulfatada a pesar de la disminución de área superficial cataliza la reacción por la acidez que contiene, dando rendimientos superiores al $80 \%$ en las mismas condiciones de reacción de la catálisis básica.

\section{Agradecimientos}

Los autores agradecen el apoyo financiero para este proyecto al PAICyT.

\section{Bibliográfia}

1. Kinast, J. A.; Tyson, K. S., "Production of biodiesel from multiple feedstocks and properties of biodiesel and biodiesel/diesel blends ${ }^{20}$. Final report; NREL: Golden, CO, (2003).

2. Ma, F. R.; Hanna, M. A., "Biodiesel production: a review ${ }^{20}$, Bioresour, Technol, 70, (1999), 1-15.

3. Haas, M. J., "The interplay between feedstock quality and esterification technology in biodiesel production "2, Lipid Technol, 16, (2004), 7-11. 
4. Talley, P., "Biodiesel "2, Render, (2004).

5. Graboski, M. S.; McCormick, R. L., "Combustion of fat and vegetable oil derived fuels in diesel engines $^{2}$, Prog. Energy Combust. Sci, 24, (1998), 125-164.

6. Zappi, M.; Hernandez, R.; Sparks, D.; Horne, J.; Brough, M.; Arora, S. M.; Motsenbocker, W. D., "A review of the engineering aspects of the biodiesel industry; Mississippi Biomass Council: Jackson ${ }^{20}$, MS, (2003); p 71.

7. Canakci, M.; Van Gerpen, J., "Biodiesel production via acid catalysis ${ }^{2,}$, Trans. ASAE, 42, (1999), 1203-1210.

8. Canakci, M.; Van Gerpen, J., "A pilot plant to produce biodiesel from high free fatty acid feedstocks $^{2}$, Trans. ASAE, 46, (2003), 945-954.

9. Canakci, M.; Van Gerpen, J., "Biodiesel production from oils and fats with high free fatty acids ${ }^{2}$, Trans. ASAE, 44, (2001), 1429- 1436.

10. Demirbas, A., "Biodiesel fuels from vegetable oils via catalytic and noncatalytic supercritical alcohol transesterifications and other methods: a survey ${ }^{2,}$ Energy Convers. Manage, 44, (2003), 2093-2109.

11. Tyagi B., Sidhpuria K., Shaik B., Jasra R.V., "Synthesis of nanocrystalline zirconia using sotgel and precipitation techniques ${ }^{2}$, Ind. Eng. Chem. Res., 45, (2006), 8643-8650.

12. Lee $\dagger$ M., Lin H., Thomas J.L., "Synthesis of zirconia with nanoporous structure by a supercritical carbon dioxide microemulsion route ${ }^{2,}$, International Journal of Applied Ceramic Technology, 7, (2010), 874-880.

13. Kazemi F., Saberi A., Malek-Ahmadi S., Sohrabi S., Rezaie H.R., Tahriri M., "A novel method for synthesis of metastable tetragonal zirconia nanopowders at low temperatures ${ }^{20}$, Ceramics Silikáty, 55, (2011), 26-30.

14. Thitsartarn W., Kawi S., "Transesterification of oil by sulfated $\mathrm{Zr}$-supported mesoporous silica ${ }^{20}$, Ind. Eng. Chem. Res., 50, (2011), 7857-7865.

15. Tyagi B., Sidhpuria K. B., Shaik B., Jasra R. V., "Effect of $\mathrm{Zr} / \mathrm{Si}$ molar ratio and sulfation on structural and catalytic properties of $\mathrm{ZrO}_{2}-\mathrm{SiO}_{2}$ mixed oxides ${ }^{20}$, J Porous Mater, 17, (2010), 699709.

16. Cao Y., Wei H., Xia Z., "Advances in microwave assisted synthesis of ordered mesoporous materials ${ }^{2}$, Trans. Nonferrous Met. Soc. China, 19, (2009), s656 - s664.

17. Corma, A.; Rodriguez, M.; Sanchez, N.; Aracil, J., "Process for the selective production of monoesters of diols and triols using zeolitic catalysts", WO9413617, (1994).

18. Perez-Pariente, J.; Diaz, I.; Mohino, F.; Sastre, E., "Selective synthesis of fatty monoglycerides by using functionalised mesoporous catalysts ${ }^{2}$, Appl. Catal., 254, (2003), 173-188.

19. Yadav, G. D.; Nair, J. J., "Sulfated zirconia and its modified versions as promising catalysts for industrial processes ${ }^{2,}$, Microporous Mesoporous Mater, 33, (1999), 1-48.

20. Wang X., Zhao J., Hou X., He Q., Tang C., "Catalytic Activity of ZrQ Nanotube arrays prepared by anodization method ${ }^{2,}$, Journal of Nanomaterials, (2012), 1-5.

21. Srivas $€$ tava, A.; Prasad, R., "Triglycerides based diesel fuels ${ }^{2}$, Renewable Sustainable Energy Rev., 4, (2000), 111-133.

22. Zhang, Y.; Dube, M. A.; McLean, D. D.; Kates, M., "Biodiesel production from waste cooking oil: 1, Process design and technological assessment ${ }^{20}$, Bioresour. Technol, 89, (2003), 1-16.

23. Zhang, Y.; Dube, M. A.; McLean, D. D.; Kates, M., "Biodiesel production from waste cooking oil: 2, Economic assessment and sensitivity analysis ${ }^{22}$, Bioresour. Technol, 90, (2003), 229-240.

24. Liu, K. S., "Preparation of FattyAcid Methyl Esters for Gas-Chromatographic Analysis of Lipids in Biological-Materials ${ }^{2}$, J. Am. Oil Chem Soc., 71, (1994), 1179-1187.

25. Fukuda, H.; Kondo, A.; Noda, H., "Biodiesel fuel production by transesterification of oils ${ }^{\nu,}$, J. Biosci. Bioeng, 92, (2001), 405-416.

26. Lutecki M., Solcova O., Werner S., Breitkopf C., "Synthesis and characterization of nanostructured sulfated zirconias ${ }^{2,}$ J Sol-Gel Sci Technol, 53, (2010), $13-20$

27. Tyagi B., Mishra M. K., Jasra R.V., "Solvent free synthesis of acetyl salicylic acid over nanocrystalline sulfated zirconia solid acid catalyst ${ }^{\circ}$, Journal of Molecular Catalysis A: Chemical, 317, (2010), 41-45

28. Saravanan K., Tyagi B. Bajaj H. C., "Esterification of caprylic acid with alcohol over nano-crystalline sulfated zirconia", J SołGel Sci Technol, 62, (2012), 13-17

29. Melo Junior C. A. R., $\dagger$ Albuquerque C. E. R., $\dagger$ Carneiro J. S. A., $\dagger$ Dariva C., $\dagger$ Fortuny M.,$\dagger$ Santos A. F., $\dagger$ Egues S. M. S, $\uparrow$ Ramos A. L. D., "Solid-acid-catalyzed esterification of oleic acid assisted by microwave heating ${ }^{2}$, Ind. Eng. Chem. Res., 49 (2010), 12135 -12139

30. Freedman, B.; Pryde, E. H.; T. L., M., "Variables affecting the yields of fatty esters from transesterified vegetable oils ${ }^{22}$, J. Am. Oil Chem. Soc., 61, (1984), 1638-1643.

31. Rattanaphra D., Harvey A., Srinophakun P., "Simultaneous conversion of triglyceride/free fatty acid mixtures into biodiesel using sulfated zirconia $^{\text {2. }}$, Top Catal, 53, (2010), 773-782

32. Srinivasan R., De Angelis R. J., Ice G., Davis B. H., "Identification of tetragonal and cubic structures of zirconia using synchrotron x-radiation

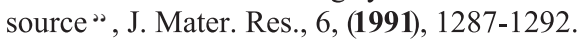

